\title{
Farinha de banana verde como ingrediente funcional em produtos alimentícios
}

\author{
Green banana flour as a functional ingredient in food products
}

\author{
Andréa dos Anjos Silva ${ }^{I^{*}}$ José Lucena Barbosa Junior ${ }^{I I}$ \\ Maria Ivone Martins Jacintho Barbosa ${ }^{\mathrm{I}}$
}

- REVISÃO BIBLIOGRÁFICA -

RESUMO

\begin{abstract}
Nos últimos anos, a banana verde tem despertado interesse do mercado consumidor, pois, além do aspecto nutricional, destaca-se a presença de compostos funcionais, em especial o amido resistente. Contudo, sua adstringência limita seu consumo e sua comercialização em forma de farinhas é a principal forma de consumo e utilização, como ingrediente funcional. $O$ objetivo do presente trabalho foi avaliar os potenciais usos da farinha de banana verde (FBV) na elaboração de produtos alimentícios e os principais métodos de obtenção, e seus efeitos sobre a qualidade fisico-química, sensorial e funcional das farinhas obtidas. Fatores como tipo de cultivar ou variedade da fruta, presença ou ausência de casca, técnica de desidratação e condições de operação do equipamento influenciam as características fisico-químicas, tecnológicas e funcionais da FBV. A FBV tem sido utilizada como ingrediente funcional na elaboração de diferentes produtos alimentícios, como pão, biscoito cookie, pré-mistura de bolo, snacks, nuggets e, sobretudo, na elaboração de massas alimentícias. De forma geral, o emprego de FBV e produtos alimenticios promove a melhoria de suas propriedades funcionais, devido ao aumento do teor de amido resistente, compostos fenólicos e da atividade antioxidante. A FBV também reduz o teor e a porcentagem de hidrólise do amido digerivel $e$, consequentemente, do indice glicêmico dos produtos, que apresentaram potencial para compor a dieta, principalmente de indivíduos diabéticos e obesos. A FBV pode ser amplamente utilizada tanto pela indústria de alimentos (pães, biscoitos, macarrão, etc.), como ingrediente na preparação de pratos prontos e na elaboração de produtos cárneos.
\end{abstract}

Palavras-chave: alimento funcional, amido resistente, Musa spp., propriedades tecnológicas, qualidade sensorial.

\section{ABSTRACT}

Recently, the market interest in green banana has raised because of its nutritional aspects, mainly due to its functional compounds as resistant starch. However, the adstringency limits green banana consumption hence it has been mostly marketed as flour or starch. The aim of this research was to evaluate the main applications of green banana flour $(G B F)$ in food products and the effects of the main utilized processes on sensorial, physicochemical and functional properties. The cultivar or variety of fruit, presence or absence of peel, type and conditions of dehydration process have influenced on the aforementioned properties of $G B F$. It was also verified that the $G B F$ has been utilized as a functional ingredient in different types of food products like breads, cookies, cake premixture, snacks, nuggets and, mainly, pastas. Altogether, the use of GBF in food products enhanced their functional properties, because it improves the resistant starch, total phenolics content and antioxidant capacity. The GBF also reduces the content and hydrolysis of digestible starch and, therefore, glycemic index of these products that show a great potential in nutrition therapy of diabetes mellitus and obesity.

Key words: functional ingredient, resistant starch, Musa spp., technological properties, sensorial quality.

\section{INTRODUÇÃO}

A banana (Musa sp.) é a fruta tropical mais consumida no mundo (SOUZA et al., 2011). Pertencente à família Musaceae, apresenta cerca de 30 espécies conhecidas do gênero Musa e mais de 700 variedades (ASMAR et al., 2013). Nutritiva, acessível à maioria da população e disponível o ano todo, é o quarto produto alimentar mais consumido no mundo (SOUZA et al., 2011). Além disso, a facilidade de propagação e o manejo fazem com que esta fruta seja a mais comercializada mundialmente, apresentando relevância econômica e social, principalmente, nas regiões tropicais (COELHO JÚNIOR, 2013).

\footnotetext{
'Programa de Pós-graduação em Ciência e Tecnologia de Alimentos (PPGCTA), Instituto de Tecnologia (IT), Universidade Federal Rural do Rio de Janeiro (UFRRJ), BR 465, km 7, 23890-000, Seropédica, RJ, Brasil. E-mail: anjosnutri@gmail.com. *Autor para correspondência.

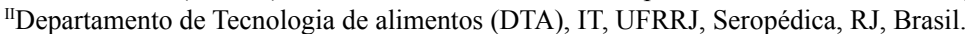


Em 2013, o Brasil produziu aproximadamente sete milhões de toneladas, sendo a Região Nordeste a principal produtora $(\sim 37 \%)$, seguida da Sudeste, com aproximadamente $32 \%$ da produção (IBGE, 2013). Em 2011, o consumo médio da fruta foi na ordem de $31 \mathrm{~kg}$ habitante ${ }^{-1} \mathrm{ano}^{-1}(\mathrm{FAO}$, 2011) e, em relação a sua importância econômica, tolerância a doenças e resistência ao frio, destacamse as seguintes variedades: Prata, Pacovan, Prata Anã, Maçã, Terra e Nanica (MOTA et al., 2000). Assim, a obtenção de farinhas se apresenta como uma excelente alternativa de geração de emprego e renda nas pequenas propriedades rurais, em que o fruto é produzido.

A boa aceitação da banana madura se deve aos seus aspectos sensoriais e nutricionais, consistindo em fonte energética, devido à presença de carboidratos, e de minerais importantes, como o potássio e vitaminas (MATSUURA et al., 2004). Entretanto, apenas nos últimos anos que o fruto verde tem despertado interesse do mercado consumidor, pois, além do seu valor nutricional, com quantidades consideráveis de vitaminas $\mathrm{B}$ e $\mathrm{C}$, bem como sais minerais, como potássio e cálcio (LEONEL et al., 2011; ASMAR et al., 2013), destacam-se a presença de amido resistente (TRIBESS et al., 2009; ALKARKHI et al., 2011), fitoesteróis, compostos fenólicos (HASLINDA et al., 2009; BERTOLINI et al., 2010; VERNAZA et al., 2011; SARAWONG et al., 2014) e capacidade antioxidante (HASLINDA et al., 2009; CHOO \& AZIZ, 2010; WANG et al., 2012; SARAWONG et al., 2014).

Entretanto, no estágio de maturação verde, a banana não é consumida, principalmente devido à típica dureza e a sua elevada adstringência, acarretada pela presença de compostos fenólicos solúveis (taninos). Essa adstringência é reduzida durante o processo de amadurecimento, quando ocorre a polimerização dessas substancias e a degradação do amido, levando ao aumento da doçura, da maciez e redução da acidez, característicos da fruta madura. Consequentemente, a obtenção de farinhas é a principal alternativa para assegurar a utilização dos frutos verdes pela indústria de alimentos (SARAWONG et al., 2014).

Segundo VERNAZA et al. (2011), a produção de farinha de banana verde (FBV) encontra ampla aplicação na indústria de alimentos, principalmente na elaboração de produtos de panificação, produtos dietéticos e alimentos infantis, sendo uma fonte de amido resistente e sais minerais, tais como potássio, cálcio, ferro, magnésio e enxofre. Além dos benefícios nutricionais, a produção de FBV contribui na redução das perdas pós-colheita, aumento do tempo de vida de prateleira e na agregação de valor à fruta (BEZERRA et al., 2013).

Dessa forma, o presente trabalho teve por objetivo avaliar os principais métodos de obtenção e seus efeitos sobre a qualidade físico-química, sensorial e funcional das farinhas obtidas e o potencial uso da FBV na elaboração de produtos alimentícios.

\section{DESENVOLVIMENTO}

Métodos de obtenção

A Legislação brasileira define farinha como "os produtos obtidos de partes comestíveis de uma ou mais espécies de cereais, leguminosas, frutos, sementes, tubérculos e rizomas por moagem e ou outros processos tecnológicos considerados seguros para produção de alimentos" (BRASIL, 2005).

$\mathrm{Na}$ obtenção da FBV, fatores como tipo de cultivar ou variedade da fruta (MOTA et al., 2000; NIMSUNG et al., 2007; HASLINDA et al., 2009), presença ou ausência de casca (BEZERRA et al., 2013), técnica de desidratação (PACHECODELAHAYE et al., 2008) e condições de operação do equipamento (TORRES et al., 2005; SARAWONG et al., 2014) podem influenciar em suas características físico-químicas, tecnológicas e funcionais.

Diversas técnicas para a obtenção da FBV têm sido observadas, conforme apresentado na tabela 1 . Esses estudos abrangem desde o uso de estufas com e sem circulação de ar (NIMSUNG et al., 2007; PACHECODELAHAYE et al., 2008; HASLINDA et al., 2009; OVANDO-MARTINEZ et al., 2009; SAIFULLAH et al., 2009; BERTOLIN et al., 2010; BORGES et al., 2010), passando por secadores em bandejas (PACHECO-DELAHAYE et al., 2008; TRIBESS et al., 2009; SANTOS et al., 2010), como também secagem assistida por outras técnicas, como a liofilização, secador de tambor duplo, micro-ondas, leito de jorro e extrusão (MOTA et al., 2000; PACHECO-DELAHAYE et al., 2008; WANG et al., 2012; BEZERRA et al., 2013; SARAWONG et al., 2014).

Diferentes cultivares podem afetar o rendimento, as características físico-químicas $\mathrm{e}$ morfológicas; propriedades reológicas, térmicas e funcionais de farinhas de banana verde (Tabela 1). SUNTHARALINGAM \& RAVINDRAN (1993) verificaram que a cultivar influenciou de maneira mais evidente o rendimento das farinhas, que não tiveram, entretanto, sua composição química afetada de forma significativa. Os autores reportaram rendimento de $31,3 \%$ para a cultivar 'Alukehel' e $25,5 \%$ para a 'Monthan'. A composição química média das cultivares foi $3,2 \%$ de proteína bruta, $1,3 \%$ de lipídeos, $3,7 \%$ de cinzas, $8,9 \%$ de fibra em detergente 
Tabela 1 -Principais cultivares de banana, métodos e condições de desidratação da farinha de banana verde (FBV).

\begin{tabular}{|c|c|c|}
\hline Cultivar & Métodos e condições de desidratação & Referência \\
\hline 'Alukehe' e 'Monthan' & $\mathrm{NE}$ & $\begin{array}{l}\text { SUNTHARALINGAM \& } \\
\text { RAVINDRAN (1993) }\end{array}$ \\
\hline Oito tipos* & Liofilização & MOTA et al. (2000) \\
\hline ‘Nanicão’' & $\begin{array}{l}\text { Extrusão/Umidade inicial da farinha }(14,3-25,7 \%) \text { e } \\
\text { temperatura }\left(108-192^{\circ} \mathrm{C}\right)\end{array}$ & TORRES et al. (2005) \\
\hline 'Kluai Khai', 'Homtong' e 'Namwa' & Estufa $50^{\circ} \mathrm{C} / 5$ horas & NIMSUNG et al. (2007) \\
\hline 'Musa paradisíaca' & $\begin{array}{l}\text { Liofilização, secador de tambor, microondas e secador } \\
\text { de bandejas }\end{array}$ & PACHECO-DELAHAYE et al (2008) \\
\hline 'Prata' & Estufa $\left(70^{\circ} \mathrm{C} / 12 \mathrm{~h}\right)$ & BORGES et al. (2009) \\
\hline 'Cavendish' & $\begin{array}{l}\text {-Secador de bandejas; -Temperaturas estudadas: } 52,55 \\
\text { e } 58{ }^{\circ} \mathrm{C} \text { e velocidade do ar de secagem: } 0,6 ; 1,0 \text { e } 1,4 \\
\mathrm{~m} . \mathrm{s}^{-1} \text {. }\end{array}$ & TRIBESS et al. (2009) \\
\hline 'Cavendish' & Estufa $\left(42^{\circ} \mathrm{C} / 72 \mathrm{~h}\right)$ & BERTOLINI et al. (2010) \\
\hline 'Cavendish' & Leito de jorro $\left(80\right.$ e $\left.90{ }^{\circ} \mathrm{C}\right)$ & BEZERRA et al. (2013) \\
\hline$' \mathrm{FBV}^{\prime} * *$ & $\begin{array}{l}\text { Extrusão/Umidade ( } 20 \text { e } 50 \%) \text { e velocidade do } \\
\text { parafuso ( } 200 \mathrm{rpm} 400 \mathrm{rpm})\end{array}$ & SARAWONG et al. (2014) \\
\hline
\end{tabular}

NE: Não especificado; FBV: Farinha de banana verde; *Variedades: Ouro Colatina, Nanica, Nanicão, Prata Anã, Prata Comum, Mysore, Maçã e Ouro da Mata; **Amostras de Chiquita doadas pela Brands International Inc, Costa Rica.

neutro, 3,8\% fibra em detergente ácido, açúcares solúveis $(2,8 \%)$, amido $(70,0 \%)$ e polissacarídeos não amiláceos $(12,0 \%)$.

Por outro lado, MOTA et al. (2000) observaram que o tipo de cultivar influenciou nas propriedades químicas, nas características morfológicas e reológicas do amido da FBV (Tabela 1). A composição química das farinhas estudadas foi de $61-76,5 \%$ de amido, 19-23\% de amilose, 2,5-3,3\% de proteína, 4-6\% de umidade, $0,3-0,8 \%$ de lipídeos, 2,6-3,5\% de cinzas e fibra total $6-15,5 \%$. Com relação aos principais resultados referentes às propriedades reológicas, observou-se que as farinhas das variedades Prata anã, Prata comum, Maçã e Ouro da Mata, durante o resfriamento, e em maiores concentrações, apresentaram o amido com maior tendência à retrogradação (setback pronunciado). Além disso, as farinhas das variedades Nanica, Nanicão e Ouro colatina apresentaram grânulos mais resistentes à fragmentação mecânica. O mesmo comportamento foi reportado por NIMSUNG et al. (2007), para farinhas de banana verde de três cultivares (Tabela 1) que apresentaram diferenças significativas na composição centesimal, nas propriedades térmicas e reológicas das farinhas avaliadas. Os autores destacaram que a farinha da cultivar 'Kluai Khai' apresentou o maior teor de proteínas, lipídeos e cinzas, temperatura de gelatinização $\left(73,96-86,18^{\circ} \mathrm{C}\right)$ e maior viscosidade de pasta que as demais cultivares estudadas.

Além do cultivar, o tipo de técnica e as condições utilizadas na obtenção também afetam a composição centesimal, características físicoquímicas, propriedades funcionais e reológicas da FBV. PACHECO-DELAHAYE et al. (2008) constataram que os diferentes métodos de desidratação estudados afetaram significativamente a composição centesimal e as propriedades funcionais e reológicas das diferentes farinhas obtidas (Tabela 1). Em especial, a secagem em tambor conferiu à farinha características de farinha pré-gelatinizada, sugerindo sua aplicação em produtos instantâneos (PACHECODELAHAYE et al., 2008).

BORGES et al. (2009) obtiveram FBV (cv. 'Prata') visando o seu aproveitamento na panificação, produtos dietéticos e alimentos infantis (Tabela 1). Os resultados indicaram que a cultivar 'Prata' apresentou elevado potencial para a obtenção de farinhas com elevado teor de amido, proteína, potássio, fósforo, magnésio, zinco e cobre.

TRIBESS et al. (2009) reportaram o efeito das condições de secagem (temperatura e velocidade do ar) no teor de amido resistente de FBV, obtidas em secador de bandeja (Tabela 1). Já em farinhas obtidas pela técnica de extrusão, a interação de importantes parâmetros do processo, como a umidade inicial da matéria-prima e velocidade do parafuso da extrusora influenciaram no teor de amilose, índice de absorção de água e de solubilidade, conteúdo de amido resistente, atividade antioxidante, fenólicos totais e nas propriedades de pasta destas farinhas (SARAWONG et al., 2014). Os autores destacaram que a combinação de maior umidade da farinha 
(50\%) com menor velocidade do parafuso (200 rpm) originou farinhas com maiores teores de amilose, amido resistente, fibras totais, índice de absorção de água e viscosidade. Além disso, apenas o índice de solubilidade em água, teor de fenólicos totais e atividade antioxidante não foram favorecidos pela condição de extrusão mencionada (SARAWONG et al., 2014). O mesmo processo foi utilizado por TORRES et al. (2005), que verificaram que o processo propiciou uma elevada degradação do amido e que as amostras apresentaram elevado grau de gelatinização em todas as condições estudadas.

Alguns estudos evidenciaram que as partes da fruta utilizadas na elaboração de farinha influenciaram a composição química, propriedades térmicas e reológicas, além do conteúdo de fitoquímicos bioativos (Tabela 1).

O aproveitamento da casca de banana verde para a produção de farinha foi estudado por BERTOLINI et al. (2010), conforme a tabela 1. Os autores reportaram que a farinha da casca apresentou maior teor de cinzas e de fitoesteróis do que a farinha controle (elaborada com a polpa da fruta), atribuído aos elevados teores de minerais e de fitoesteróis encontrados na casca da fruta. A farinha da casca apresentou menor viscosidade e maior temperatura de gelatinização do que as elaboradas com a polpa da fruta (Tabela 1). Efeito similar foi reportado por BEZERRA et al. (2013), que estudaram farinhas elaboradas com banana verde com e sem casca pela técnica de secagem em leito de jorro (Tabela 1). A presença da casca da banana influenciou significativamente na composição química, na cor, na viscosidade da pasta e tamanho de partícula da farinha, justificado pela presença de material fibroso da casca. Em especial, a farinha elaborada com a polpa e a casca da banana apresentou menor teor de lipídeos, cinzas e fibras totais, e teores mais elevados de carboidratos, amido total e resistente do que a elaborada apenas com a polpa da fruta.

O uso da farinha de banana verde como ingrediente funcional Segundo a Agência Nacional de Vigilância Sanitária, o alimento ou ingrediente que alegar propriedades funcionais, além de funções nutricionais básicas, quando se tratar de nutriente, deverá produzir efeitos metabólicos e/ou fisiológicos e ou efeitos benéficos à saúde, devendo ser seguro para o consumidor sem supervisão médica (BRASIL, 1999).

$\mathrm{O}$ amido resistente, que pode ser definido como parte da molécula do amido que resiste à degradação pela $\alpha$-amilase e, consequentemente, à digestão no intestino delgado, servindo de substrato para bactérias no cólon de indivíduos saudáveis, pode ser, portanto, considerado um composto funcional (RAMOS et al., 2009; TRIBESS et al.,2009).

Quanto à facilidade de absorção do amido pelo intestino delgado, os alimentos podem ser classificados como fontes de: amido de rápida absorção (ARA); amido de lenta absorção (ALA); ou amido resistente (AR). Cerca de $75 \%$ em peso da farinha de banana verde (FBV) são constituídos de amido, dos quais $3 \%$ são ARA, $15 \%$ ALA e $57 \%$ são AR (ZHANG et al., 2005). A inclusão do amido resistente na dieta humana tem sido associada à redução dos níveis de colesterol LDL (lipoproteína de baixa densidade), assim como a redução de triglicerídeos na hiperlipidemia. Não sendo digerido no intestino delgado, o amido resistente também pode servir de substrato para o crescimento de microrganismos probióticos, atuando como potencial agente prebiótico (WALTER et al., 2005).

Além disso, a metabolização desse tipo de carboidrato pelos microrganismos via fermentação resulta na produção de ácidos graxos de cadeia curta, como acetato, propionato e butirato; gases carbônico, hidrogênio e, em alguns indivíduos, metano; e diminuição do pH do cólon (WALTER et al., 2005; BERTOLIN et al., 2010). Segundo BEZERRA et al. (2013), a ingestão de AR na Europa e na América Latina ainda é baixo (3-6g dia $\left.{ }^{-1}\right)$ em relação ao consumo em outras regiões, como a Ásia (8-19g dia $\left.{ }^{-1}\right)$. Assim, verifica-se um elevado potencial para o aumento da ingestão desse nutriente nos produtos alimentícios.

$\mathrm{O}$ teor de $\mathrm{AR}$ nas farinhas varia em função do tipo de cultivar, presença ou ausência de casca, da técnica e da condição de obtenção (RAMOS et al., 2009; BEZERRA et al., 2013; SARAWONG et al., 2014), podendo chegar a até $40 \%$ em peso de FBV (ZHANG et al., 2005; RAMOS et al., 2009).

Em função do seu elevado teor de amido resistente e de sua ação redutora sobre o índice glicêmico (IG), diversos estudos têm relatado o uso da FBV na elaboração de vários produtos alimentícios (Tabela 2).

Pães formulados com FBV $(65,08 \%)$ apresentaram menor índice glicêmico (IG), significativamente menor no pão controle $(81,88 \%)$, sugerindo que este produto pode ser utilizado como alternativa para alimentação de indivíduos que apresentam dieta com restrição calórica (JUAREZGARCIA et al., 2006). Além disso, os autores reportaram um aumento significativo dos teores de umidade, cinzas, proteínas, amido total, amido resistente e fibra dietética, quando comparado ao controle $(100 \% \mathrm{FT})$. O mesmo resultado foi observado para macarrão (Tabela 2) elaborado com substituição parcial da farinha de trigo por FBV 
Tabela 2 - Produtos elaborados com farinha de banana verde (FBV) e suas formulações.

\begin{tabular}{|c|c|c|}
\hline Produtos & Formulações & Referência \\
\hline Pré mistura para pão & -FT: FBV (90:10). & JUAREZ-GARCIA et al. (2006) \\
\hline Biscoito tipo cookie & $-0 \%$ (controle), 10,20 e $30 \%$ de FBV. & FASOLINI et al. (2007) \\
\hline Espaguete & $\begin{array}{l}\text {-100\% de sêmola de trigo duro (controle) e misturas de sêmola: FBV, } \\
\text { nas proporções } 85: 15,70: 30 \text { e 55:45. }\end{array}$ & OVANDO-MARTINEZ et al. (2009) \\
\hline Macarrão & $\begin{array}{l}-90 \mathrm{~g} \text { de FT, } 50 \mathrm{~mL} \text { água destilada, } 1 \mathrm{~g} \text { de sal, } 10 \mathrm{~g} \text { de FBV e } 1 \mathrm{~mL} \text { água } \\
\text { alcalina }(K A N S U) \text {. }\end{array}$ & SAIFULLAH et al. (2009) \\
\hline Pré mistura para bolo & -Substituição da FT por $60 \%$ de FBV. & BORGES et al. (2010) \\
\hline Macarrão & $-30 \%$ de FBV em substituição a FT. & CHOO \& AZIZ (2010) \\
\hline Macarrão instantâneo & -Adição de $10 \%$ de FBV. & VERNAZA et al. (2011) \\
\hline Snacks & $\begin{array}{l}\text {-Snacks de mandicoca: } 0,10,20,30,40,50 \mathrm{gde} F B V / 100 \mathrm{~g} \text { de amido } \\
\text { de mandioca; } \\
\text { Snacks de peixe: } 0,5,10,15,20,25 \mathrm{~g} \text { de FBV/100g amido de } \\
\text { mandioca }\end{array}$ & WANG et al (2012) \\
\hline Biscoito tipo cookie & -Proporções de $0 \%$ (controle), $15 \%, 30 \%$ e $50 \%$ p/p de FBV & AGAMA-ACEVEDO et al. (2012) \\
\hline Macarrão & $\begin{array}{l}-47,5 \% \text { de FBV; } 31,5 \% \text { de ovos; } 16,4 \% \text { de água; } 2,5 \% \text { de goma guar e } \\
2,5 \% \text { de goma xantana. }\end{array}$ & ZANDONADI et al. (2012) \\
\hline Nuggets de frango & -Adição de $0 \%$ (controle), 3\%, 4\% e 5\% de FBV. & KUMAR et al. (2013). \\
\hline
\end{tabular}

FBV: Farinha de banana verde; FT: Farinha de trigo.

(SAIFULLAH et al., 2009). O mesmo produto foi estudado por CHOO \& AZIZ (2010) e, conforme o esperado, o macarrão formulado com FBV apresentou menores índices glicêmico e de digestibilidade de carboidratos (Tabela 2). Além disso, as amostras adicionadas de FBV não apresentaram diferenças significativas na aceitação sensorial em comparação às amostras controle. Biscoitos formulados com diferentes \% de FBV (Tabela 2) apresentaram redução do \% de hidrólise do amido digerível e, por conseguinte, do IG, refletindo na redução da absorção de glicose, podendo ser indicado para compor a dieta, principalmente de indivíduos diabéticos e obesos (AGAMA-ACEVEDO et al., 2012).

A adição de FBV também tem contribuído para melhoria das propriedades funcionais de vários produtos. OVANDO-MARTINEZ et al. (2009) verificaram que a adição de FBV aumentou os teores de amido total e resistente de compostos fenólicos e a atividade antioxidante de espaguetes (Tabela 2). Em biscoito tipo snacks, WANG et al. (2012) reportaram que a adição de FBV melhorou o valor nutricional, destacando-se os teores de fibra, minerais, polifenóis e a capacidade antioxidante das amostras (Tabela 2).

Macarrão instantâneo (Tabela 2) com propriedades funcionais foi desenvolvido por VERNAZA et al. (2011), que observaram redução significativa dos teores de gordura e de amido resistente, nas amostras adicionadas de FBV.

A utilização da FBV também foi testada em produtos cárneos, na formulação de nuggets de frango (Tabela 2) por KUMAR et al. (2013). Os autores destacaram que não foi observada diferença sensorial significativa entre a aceitação global, o aroma, aparência, textura e a suculência do controle e as amostras de nuggets formuladas com até 3\% de FBV.

Alguns estudos têm relatado que o uso da FBV não tem afetado a qualidade microbiológica (BORGES et al., 2010) e sensorial (FASOLIN et al., 2007; CHOO \& AZIZ, 2010; ZANDONADI et al., 2012) dos produtos. Com relação à qualidade microbiológica, a adição de FBV em pré-mistura de bolo não apresentou alterações microbiológicas durante 120 dias de armazenamento, estando em conformidade com a Legislação, segundo BORGES et al. (2010).

Biscoitos tipo cookie (Tabela 2), formulados com substituição parcial da farinha de trigo por FBV foram desenvolvidos por FASOLINI et al. (2007). O teste de aceitação dos biscoitos indicou que não houve diferença significativa entre as diferentes formulações e o controle, com exceção para o biscoito com $30 \%$ de FBV, que apresentou menor aceitação entre as crianças.

Macarrão sem glúten (Tabela 2), elaborado com farinha de banana verde, foi desenvolvido por ZANDONADI et al. (2012). De forma geral, este produto apresentou maior aceitação sensorial $(84,5 \%$ provadores celíacos e $61,2 \%$ provadores sem DC) do que amostras padrão (53,6\% para indivíduos sem DC). Não houve diferença significativa entre as amostras com farinha de banana e o padrão em termos de aparência, aroma, sabor e qualidade global. 


\section{CONCLUSÃO}

A literatura disponível sugere que a farinha de banana verde apresenta propriedades que lhe conferem um grande potencial na indústria de alimentos. O baixo custo e alta disponibilidade da fruta, na forma in natura, oferecem um mercado favorável aos produtores de FBV, sobretudo pequenos produtores rurais familiares. Podem ser amplamente utilizadas tanto pela indústria de alimentos (pães, biscoitos, macarrão, etc.) como ingrediente na preparação de pratos prontos, tanto pelas conhecidas características das farinhas de banana tradicionais, quanto por serem uma excelente fonte de amido resistente, compostos fenólicos e antioxidantes, além de apresentarem um potencial como fonte de fitoesteróis. Apesar do processo de obtenção ser bastante tradicional, algumas tecnologias alternativas têm sido observadas. Contudo, mesmo na secagem por convecção tradicional, há controvérsia na definição das condições de operação e na qualidade das farinhas, Portanto, ainda são necessários esforços na melhor compreensão do efeito dos processos nos aspectos sensoriais, físico-químicos e nutricionais das farinhas de banana verde.

\section{REFERÊNCIAS}

AGAMA-ACEVEDO, E. et al. Starch digestibility and glycemic index of cookies partially substituted with unripe banana flour. LWT-Food Science and Technology, v.46, p.177-182, 2012. Disponível em: < http://www.sciencedirect.com/science/article/pii/ S0023643811003367>. Acesso em: 23 fev. 2015. doi: 10.1016/j. lwt.2011.10.010.

ALKARKHI, A.F. et al. Comparing physicochemical properties of banana pulp and peel flour prepared from green and ripe fruits. Food Chemistry, v.129, p.312-318, 2011. Disponível em: <http:// www.sciencedirect.com/science/article/pii/S0308814611006261>. Acesso em: 23 fev. 2015. doi: 10.1016/j.foodchem.2011.04.060.

ASMAR, S. A. et al. Changes in leaf anatomy and photosynthesis of micropropagated banana plantlets under silicon sources. Scientia Horticulturae, v. 161, p. 328-332, 2013. Disponível em: <http:// www.sciencedirect.com/science/article/pii/S0304423813003713>. Acesso em: 12 jun. 2014. doi: 10.1016/j.scienta.2013.07.021.

BERTOLINI, A.C. et al. Rheological and functional properties of flours from banana pulp and peel. Starch/Starke, v.62, p .277-284, 2010. Disponível em: $<\mathrm{http}$ ://onlinelibrary.wiley.com/doi/10.1002/ star.200900216/pdf>. Acesso em: 25 fev.2015.

BEZERRA C.V. et al. Green banana (Musa cavendishii) flour obtained in spouted bed - Effect of drying on physicochemical, functional and morphological characteristics of the starch. Industrial Crops and Products, v.41, p.241-249, 2013. Disponível em: <http://www.sciencedirect.com/science/article/ pii/S0926669012002257>. Acesso em: 03 fev. 2014. doi: 10.1002/ star.200900216.
BORGES, A. M. et al. Caracterização da farinha de banana verde. Ciência e Tecnologia de Alimentos, v. 29, n. 2, p. 333-339, 2009. Disponível em: $<$ http://www.scielo.br/readcube/epdf.php $>$. Acesso em: 03 fev. 2014. doi: 10.1590/S0101-20612009000200015

BORGES, A.M. et al. Estabilidade da pré-mistura de bolo com $60 \%$ de farinha de banana verde. Ciência e Agrotecnologia, v.34, n.1, p.173-181, 2010. Disponível em: <http://www.scielo.br/ scielo.php?script=sci_arttext\&pid=S1413-70542010000100022>. Acesso em: 03 fev. 2014. doi:10.1590/S1413-70542010000100022.

BRASIL. Resolução n.19, de 30 de Abril de 1999. Regulamento técnico de procedimentos para registro de alimento com alegação de propriedades funcionais e ou de saúde em sua rotulagem. Seção 1, p.11. 1999c. Disponível em: <http://portal. anvisa.gov.br/wps/wcm>. Acesso em: 04 fev. 2014.

BRASIL. Resolução n.263, de 22 de setembro de 2005. Regulamento técnico para misturas para o preparo de alimento e alimentos pronto para o consumo. Disponível em: $<$ http://www.anvisa.gov.br/alimentos/legis/especifica/regutec. htm>. Acesso em: 22 jan. 2013.

CHOO, C.L.; AZIZ, N.A.A. Effects of banana flour and $\beta$-glucan on the nutritional and sensory evaluation of noodles. Food Chemistry, v.119, p.34-40, 2010. Disponível em: <http://www. sciencedirect.com/science/article/pii/S0308814609006220>. Acesso em: 05 fev. 2015. doi: 10.1016/j.foodchem.2009.05.004.

COELHO JÚNIOR, L.M.C. Concentração regional do valor de produção da banana do Paraná, Brasil (1995 a 2010). Ciência Rural, v.43, n.12, p.2304-2310, 2013. Disponível em: <http:// www.scielo.br/pdf/cr/v43n12-1004.pdf>. Acesso em: 24 fev. 2014

FAO (FOOD AND AGRICULTURE ORGANIZATION). Consumo. 2011. Disponível em: <http://faostat.fao.org/site/609/ DesktopDefault.aspx?PageID=609\#ancor $>$. Acesso em: jun. 2014.

FASOLIN, L.H. et al. Biscoitos produzidos com farinha de banana verde: avaliações química, física e sensorial. Ciência e Tecnologia de Alimentos, v.27, n.3, p.524-529, 2007. Disponível em: <http://www.scielo.br/scielo.php?pid=S010120612007000300016\&script=sci_arttext $>$. Acesso em: 14 fev. 2014. doi: 10.1590/S0101-20612007000300016.

HASLINDA, W.H. etal.Chemical composition and physicochemical properties of green banana (Musa acuminate x Balbisianacolla cv. 'Awak') flour. International Journal of Food Sciences and Nutrition, v.60, n.4, p.232-239, 2009. Disponível em: < http:// informahealthcare.com/doi/abs/10.1080/09637480902915525>. Acesso em: 12 fev. 2014. doi: 10.1080/09637480902915525.

IBGE (INSTITUTO BRASILEIRO DE GEOGRAFIA E ESTATÍTICA). Levantamento Sistemático da Produção Agrícola. Pesquisa Mensal de Previsão e Acompanhamento das Safras Agrícolas no Ano Civil. Disponível em: <http://www.ibge. gov.br>. Acesso em: $22 \mathrm{dez} .2013$.

JUAREZ-GARCIA, E. et al. Composition, digestibility and application in bread making of banana flour. Plant Foods for Human Nutrition, v.61, p.131-137, 2006. Disponível em: <http:// www.ncbi.nlm.nih.gov/pubmed/17048100>. Acesso em: 05 fev. 2014. doi: 10.1007/s11130-006-0020-x.

KUMAR, V. et al. Quality and storability of chicken nuggets formulated with Green and soybean hulls flours. Journal 
Food Science Technology, v.50, n.6, p.123-134, 2013. Disponível em: $<$ http://link.springer.com/article/10.1007\%2 Fs13197-011-0442-9>. Acesso em: 11 fev. 2014. doi: 10.1007/ s13197-011-0442-9.

LEONEL, M. et al. Extração e caracterização do amido de diferentes genótipos de bananeira. Revista Brasileira de Fruticultura, v.33, p.599-605, 2011. Disponível em: <http://www.scielo.br/pdf/rbf/ v33nspe1/a82v33nspe1.pdf>. Acesso em: 03 mar. 2015.

MATSUURA, F.C.A.U.et al. Marketing de banana: preferências do consumidor quanto aos atributos de qualidade dos frutos. Revista Brasileira de Fruticultura, v.26, p.48-52, 2004. Disponível em: $<$ http:// www.scielo.br/pdf/rbf/v26n1/a14v26n1>. Acesso em: 23 abr. 2014.

MOTA, R.V. et al. Composition and functional properties of banana flour from different varieties. Starch/starke, v.52, p.63-68, 2000. Disponível em: <http://onlinelibrary.wiley.com/ doi/10.1002/(SICI) 1521-379X(200004)52:2/3\%3C63::AIDSTAR63\%3E3.0.CO;2-V/pdf>. Acesso em: 06 jul. 2014. doi: 0038-9056/2000/0202-0063.

NINSUNG, P. et al. Compositions, morphological and thermal properties of green banana flour and starch. Kasetsart Journal, v.41, p.324-330, 2007. Disponível em: <http://www.thaiscience. info/journals/Article>. Acesso em: 05 mar. 2015.

OVANDO-MARTINEZ, M. et al. Unripe banana flour as an ingredient to increase the indigestible carbohydrates of pasta. Food Chemistry, v.113, p.121-126, 2009. Disponível em: $<\mathrm{http}: /$ www.sciencedirect.com/science/article/pii/S0308814608008583>. Acesso em: 3 jun. 2015. doi: 10.1016/j.foodchem.2008.07.035.

PACHECO-DELAHAYE, E. et al. Production and characterization of unripe plantain (Musa paradisiacal L.) flours. Interciência v.33, n.4, p.290-296, 2008. Disponível em: <http://www. http:// www.scielo.org.ve/pdf/inci/v33n4/art11.pdf $>$. Acesso em: 03 jun. 2014. doi: 0378-1844/08/04/290-06.

RAMOS, D.P. et al. Resistant starch in Green banana flour. Brazilian Journal of Food and Nutrition, v.20, n.3, p.479-483, 2009. Disponível em: <http://serv-bib.fcfar.unesp.br/seer/index.php/ alimentos/article/view/1151/846>. Acesso em: 06 mai. 2014.

SAIFULLAH, R. et al. Utilization of Green flour as a functional ingredient in yellow noodle. International Food Research Journal, v.16, p.373-379, 2009. Disponível em: <http://ifrj.upm. edu.my/16\%20(3)\%202009/10[1]\%20Saifullah.pdf>. Acesso em: 04 abr. 2014

SANTOS, J.C. et al. Processamento e avaliação da estabilidade da farinha de banana verde. Exacta, v. 8, n.2, p.219-224, 2010. Disponível em: $<$ http:// www.redalyc.org/articulo.oa?id=81016917011>. Acesso em: 07 abr. 2014.

SARAWONG, C. et al. Effect of extrusion cooking on the physicochemical properties, resistant starch, phenolic content and antioxidant capacities of green banana flour. Food Chemistry, v.143, p.33-39, 2014. Disponível em: <http://www.sciencedirect.
com/science/article/pii/S0308814613010108>. Acesso em: 06 jun. 2014. doi: 10.1016/j.foodchem.2013.07.081.

SOUZA, M. E. et al. Crescimento e produção de genótipos de bananeiras em clima subtropical. Ciência Rural, v.41, n.4, p.581591, 2011. Disponível em: <http://www.scielo.br/pdf/cr/v41n4/ a924cr4241.pdf>. Acesso em: 02 jul. 2014.

SUNTHARALINGAM, S.; RAVINDRAM, G. Physical and biochemical properties of green banana flour. Plant Food for Human Nutrition, v.43, p.19-27, 1993. Disponível em: <http://www.ncbi. nlm.nih.gov/pubmed/8385333>. Acesso em: 02 mai. 2014.

TORRES, L. L. G. et al. Efeito da umidade e da temperatura no processamento de farinha de farinha de banana verde (Musa acuminata, grupo AAA) por extrusão termoplástica. Boletim CEPPA, v.23, n.2, p.273-290, 2005. Disponível em: <http://ojs. c3sl.ufpr.br/ojs/index.php/alimentos/article/view/4488/3509>. Acesso em: 23 abr. 2014. doi: 10.5380/cep.v23i2.4488.

TRIBESS, T.B. et al. Thermal proprieties and resistant starch content of green banana flour (Musa cavendishii) produced at different drying conditions. LWT- Food Science and Technology, v.42, p.1022-1025, 2009. Disponível em: <http://www. sciencedirect.com/science/article/pii/S002364380900005X $>$. Acesso em: 25 fev. 2015. doi: 10.1016/j.lwt.2008.12.017.

VERNAZA, G.V. et al. Addition of green banana flour to instant noodles: Rheological and technological properties. Ciências e Agrotecnologia, v.35, n.6, p.1157-1165, 2011. Disponível em: <http://www.scielo.br/scielo.php?script=sci arttext\&pid=S1413-70542011000600016>. Acesso em: $25 \mathrm{fev}$. 2015. doi: 10.1590/S1413-70542011000600016.

WALTER, M. et al. Amido resistente: características físicoquímicas, propriedades fisiológicas e metodológicas de quantificação. Ciência Rural, v.35, n.4, p.974-980, 2005. Disponível em: <http://www.scielo.br/pdf/cr/v35n4/a41v35n4>. Acesso em: 23 out. 2014

WANG, Y. et al. Influence of green banana flour substitution for cassava starch on the nutrition color, texture and sensory quality in two types of snacks. LWT-Food Science and Technology, v.47, p.175-182, 2012. Disponível em: <http://www.sciencedirect.com/ science/article/pii/S002364381100404X>. Acesso em: 23 out. 2014. doi: 10.1016/j.1wt.2011.12.011.

ZANDONADI, R.P. et al. Green banana pasta: an alternative for gluten-free diets. Journal of the academy of nutrition and dietetics, v.112, n.7, p.1068-1072, 2012. Disponível em: <http:// www.sciencedirect.com/science/article/pii/S221226721200473X >. Acesso em: 05 mai. 2014. doi: 10.1016/j.jand.2012.04.002.

ZHANG, P. et al. Resistant banana starch: production, physicochemical properties, and digestibility -A review. Carbohydrate Polymers, v.59, p.443-458, 2005. Disponível em: $<$ http://www.ncbi.nlm.nih.gov/pubmed/21214175>. Acesso em: 30 jun. 2014. doi: 10.1021/jf1035004. 